Ciência Rural, Santa Maria, v.43, n.2, p.219-224, fev, 2013

ISSN 0103-8478

\title{
Crescimento e desenvolvimento de pessegueiros 'Chimarrita' e 'Maciel' sobre diferentes porta-enxertos e locais de cultivo
}

\author{
Growth and development of 'Chimarrita' and 'Maciel' peach trees on different rootstocks and \\ cultivating areas
}

\author{
Simone Padilha Galarça ${ }^{I^{*}}$ José Carlos Fachinello $^{\mathrm{I}}$ Débora Leitzke Betemps ${ }^{\mathrm{II}}$ \\ Alexandre Hoffmann ${ }^{\text {III }}$ Gilmar Arduino Bettio Marodin ${ }^{\text {IV }}$ Aloir Pretto $^{\text {I }}$ Fabrício Silva Nunes ${ }^{\text {IV }}$ \\ Felipe Pereira Dias ${ }^{\mathrm{IV}}$
}

\section{RESUMO}

Objetivou-se com o presente trabalho avaliar a influência dos porta-enxertos no que se refere ao crescimento vegetativo e desenvolvimento dos pessegueiro 'Chimarrita' $e$ 'Maciel' em três locais de cultivo. O experimento foi desenvolvido em três locais de cultivo: 1 (Embrapa Uva e Vinho), 2 (EEA UFRGS) e 3 (CAP UFPel), em três anos. As cultivares de pessegueiro utilizadas foram: 'Chimarrita' $e$ 'Maciel' enxertadas sobre seis porta-enxertos ('Aldrighi', 'Capdeboscq', 'Flordaguard', 'Nemaguard', 'Okinawa'e Umezeiro), no espaçamento $1,5 \times 5,0 m$ e conduzidas no sistema em " $V$ ". Foram avaliados: volume de copa, diâmetro de tronco, massa fresca da poda, produção por planta acumulada, comprimento, espessura, gemas floríferas e densidade florífera do ramo produtivo. Também foi avaliado de forma visual o ponto de enxertia. O porta-enxerto Umezeiro induziu menor vigor nos três locais de cultivo estudados e na média dos três anos. Ele pode ser indicado para utilização sob 'Maciel' nas condições edafoclimáticas do local 3. No local 1 o porta-enxerto, 'Nemaguard' possui alto vigor e maior produção acumulada, sendo o mais indicado. Já para o local 2, os porta-enxertos 'Capdeboscq', 'Okinawa' e 'Flordaguard' são os mais indicados. Os porta-enxertos afetam as características de crescimento e desenvolvimento das cultivares 'Chimarrita' e 'Maciel', o vigor destas é menor sobre o portaenxerto Umezeiro nos três locais de cultivo. Todos os portaenxertos podem ser indicados para os três locais de cultivo, com exceção do Umezeiro, que somente pode ser indicado em combinação com a cultivar 'Maciel' no local 3.

\begin{abstract}
The objective of this study was to evaluate the influence of rootstocks in relation to vegetative growth and development of 'Chimarrita' and 'Maciel' peach trees in three places of cultivation. The experiment was conducted in three cultivating areas: first (Embrapa Grape and Wine), second (EEA UFRGS) and third (CAP UFPel), in three years. Peach trees cultivars used were: 'Chimarrita' and 'Maciel,' grafted on six rootstocks ('Aldrighi' 'Capdeboscq,' 'Flordaguard,' 'Nemaguard,' 'Okinawa' and Mume), with a spacing of $1.5 \mathrm{~m}$ by $5.0 m$ and conducted to a "V" system. It was evaluated: canopy volume, trunk diameter, pruning fresh weight, cumulative yield, length, thickness, flower bud and flower density of productive branch. It was also visually assessed the grafting point. The Mume rootstocks induced less vigor in the three cultivation areas studied, and in the average of three years. And it can be indicated for use on 'Maciel' at edaphoclimatic conditions of the third area. In the first area, the 'Nemaguard' rootstock has high vigor and greater cumulative production, being the best. As for the second area, the 'Capdeboscq,' 'Okinawa' and 'Flordaguard' rootstocks are the most suitable. It can be concluded that rootstocks affect the growth and development characteristics of 'Chimarrita' and 'Maciel' cultivars, the vigor of these is lower on the Mume rootstock in the three cultivating areas. All rootstocks can be indicated for the three areas of cultivation, with the exception of Mume, which can only be indicated in combination with 'Maciel' cultivar in the third area.
\end{abstract}

Key words: Prunus sp., vigor, divergence.

Palavras-chave: Prunus sp., vigor, divergência.

IPrograma de Pós- graduação em Agronomia, Fruticultura de Clima Temperado, Departamento de Fitotecnia, Universidade Federal
de Pelotas (UFPel), CP 354, 96010-900, Pelotas, RS, Brasil. E-mail: sgalarca@ superig.com.br. *Autor para correspondência. de Pelotas (UFPel), CP 354, 96010-900, Pelotas, RS, Brasil. E-mail: sgalarca
"Universidade Federal da Fronteira Sul (UFFS), Laranjeiras do Sul, PR, Brasil.

"IIEmbrapa Uva e Vinho, Bento Gonçalves, RS, Brasil.

${ }^{\text {IV } U n i v e r s i d a d e ~ F e d e r a l ~ d o ~ R i o ~ G r a n d e ~ d o ~ S u l ~(U F R G S), ~ P o r t o ~ A l e g r e, ~ R S, ~ B r a s i l . ~}$ 


\section{INTRODUÇÃO}

Devido à alta demanda do mercado de produtos hortícolas e frutícolas, as espécies são cultivadas sob diversas condições ambientais que podem proporcionar estresse, influenciando seu crescimento e consequentemente o desenvolvimento. Essas condições incluem a temperatura, climas secos ou úmidos, baixa ou alta radiação, solos com boa ou má drenagem, assim como fertilidade natural dos solos, entre outros (MARTÍNEZ-BALESTA et al., 2010). Para enfrentar essas adversidades, uma das alternativas é a utilização de diferentes porta-enxertos, entretanto, fazse necessário conhecer como este se expressa em diferentes regiões de cultivo.

Os porta-enxertos são responsáveis pela absorção de água e nutrientes, resistência a patógenos do solo e tolerância a estresses ambientais (ABDELMAGEED \& GRUDA 2009). Eles influenciam nos atributos agrícolas mais importantes para as plantas, como o vigor, produção, floração e qualidade das frutas (DICHIO et al., 2004; JIMÉNEZ et al., 2007; HERNÁNDEZ et al., 2010).

Um bom porta-enxerto deve ser compatível com as cultivares copa, resistente e ou tolerante a pragas e doenças e adaptado a uma ampla gama de tipos de solo e condições climáticas (DICHIO et al., 2004). Existem, na base mundial, diferentes tipos de porta-enxertos usados para Prunus, porém cada um tem determinado conjunto de vantagens e limitações para a adaptação a diferentes regiões geográficas (HERNÁNDEZ et al., 2010).

No Brasil, mais especificamente no Rio Grande do sul, os estudos sobre porta-enxertos ainda são incipientes, principalmente para pessegueiro. Já foram realizados por DE ROSSI et al. (2004), que avaliaram pessegueiro 'Granada' sobre diferentes portaenxertos, PICOLOTTO et al. (2009), que avaliaram pessegueiros 'Chimarrita' sobre diferentes portaenxertos. Esses estudos são importantes, porque, segundo RATO et al. (2008), os produtores de cada região devem ter informações sobre os atributos e as limitações dos porta-enxertos específicos, bem como os efeitos de solo e clima da referida região.

Com base no exposto, objetivou-se com o presente trabalho avaliar a influência dos porta-enxertos no que se refere ao crescimento vegetativo e desenvolvimento dos pessegueiro 'Chimarrita' e 'Maciel' em três locais de cultivo.

\section{MATERIAL E MÉTODOS}

O experimento foi conduzido em três campos experimentais. Local 1: Embrapa Uva e Vinho (Embrapa
Uva e Vinho), localizada no município de Bento Gonçalves/RS. Local 2: Estação Experimental Agronômica da Universidade Federal do Rio Grande do Sul (EEA UFRGS), localizada em Eldorado do Sul/ RS a $50 \mathrm{~km}$ de Porto Alegre. Local 3: Centro Agropecuário da Palma (CAP UFPel), pertencente à Universidade Federal de Pelotas, localizado no município de Capão do Leão/RS. As cultivares copa utilizadas foram: Chimarrita e Maciel sobre os portaenxertos: 'Aldrighi', 'Capdeboscq', 'Flordaguard', 'Nemaguard', 'Okinawa' e 'Umezeiro'. Os portaenxertos foram provenientes de sementes.

$\mathrm{O}$ sistema de condução foi em "V" e o espaçamento entre linhas é de $5 \mathrm{~m}$ e entre plantas $1,5 \mathrm{~m}$. O delineamento experimental utilizado foi em casualização por blocos com três repetições, cinco plantas por unidade experimental, sendo avaliadas três plantas e as demais consideradas como bordadura. Em esquema unifatorial (porta-enxertos) para cada copa 'Chimarrita' e 'Maciel' e cada local de cultivo.

As variáveis avaliadas foram: volume de copa $\left(\mathrm{m}^{-3}\right)$ (VC), conforme descrito por De ROSSI (2004); diâmetro do tronco (mm) (DT); massa fresca do material vegetal retirado na poda $(\mathrm{kg})(\mathrm{MFP})$, realizada em cada ano (total de três podas por ano), produção por planta acumulada $\left(\mathrm{kg} \mathrm{pl}^{-1}\right)$ (PPA), total de três anos. Foi avaliado o comprimento $(\mathrm{cm})(\mathrm{C})$, espessura $(\mathrm{mm})(\mathrm{E})$, número de gemas floríferas (GF) e densidade florífera (no flores $25 \mathrm{~cm}^{-1}$ ) (DF) (locais 1 e 3). Também foi avaliado de forma visual o ponto de enxertia. Para as avaliações, foi realizada a média dos três anos (2009, 2010e 2011).

Para verificação dos efeitos do porta-enxerto sobre as cultivares copas, nos diferentes locais, realizou-se a análise de variância. Quando significativa, procedeu-se o agrupamento de médias univariado pelo teste de Scott e Knott com nível de significância de $5 \%$, utilizando o programa estatístico Genes (CRUZ, 2001). Esse procedimento foi utilizado para reduzir a ambiguidade nos resultados e facilitar a interpretação dos dados.

\section{RESULTADOS E DISCUSSÃO}

No local 1, (Embrapa Uva e Vinho) houve diferença significativa para as variáveis volume de copa (VC), diâmetro de tronco (DT), massa fresca retirada na poda (MFP) e produção por planta acumulada (PPA) para o pessegueiro 'Chimarrita' sobre diferentes portaenxertos. E DT, MFP e PPA para o pessegueiro 'Maciel' sobre diferentes porta-enxertos. Para 'Chimarrita', o porta-enxerto Umezeiro induziu menor VC, DT, MFP, PPA. Para 'Maciel', o porta-enxerto Umezeiro induziu 
menor DT e MFP e uma das menores PPA, enquanto que 'Nemaguard' proporcionou maior PPA ao pessegueiro 'Maciel' (Tabela 1). Na avaliação visual do ponto de enxertia, foi possível observar um intumescimento nas combinações dos pessegueiros 'Chimarrita' e 'Maciel' com Umezeiro.

A partir dos resultados observados, podese inferir que o porta-enxerto Umezeiro proporciona menor vigor aos pessegueiros 'Chimarrita' e 'Maciel' no local 1, (Embrapa Uva e Vinho) (Tabela 1). Esse fato pode ser atribuído a certo grau de incompatibilidade observado através do intumescimento do ponto de enxertia ou ainda pelo fato de esse porta-enxerto, segundo MAYER \& PEREIRA (2006), ser considerado com possibilidades de sucesso como ananizante para pessegueiro na formação de pomares de alta densidade. DE ROSSI et al. (2004), também encontraram que o porta-enxerto Umezeiro induz menor vigor para 'Granada', porém não foram constatados sintomas de incompatibilidade.

A questão da compatibilidade é bastante complexa, segundo USENIK et al. (2006), e ainda não é bem estudada. Para que algumas substâncias auxiliem na detecção da incompatibilidade, elas devem estar presentes tanto nas cultivares copa como no portaenxerto, porém, segundo os mesmos autores, substâncias produzidas na região da enxertia, como a concentração dos fenóis, podem representar um indício da incompatibilidade.

Já o fato de o porta-enxerto Umezeiro ter proporcionado uma das mais baixas produções acumuladas durante os três anos, tanto para 'Chimarrita' quanto para 'Maciel', deve-se ao fato de este proporcionar plantas menores, produzindo menor quantidade de frutas, porém tem a vantagem da possibilidade de adensamento em relação aos demais porta-enxertos. Nas condições do experimento, o portaenxerto que se destacou em PPA, tanto para 'Chimarrita' como para 'Maciel' foi o 'Nemaguard'.

No local 2 (EEA UFRGS), para o pessegueiro 'Chimarrita', novamente o porta-enxerto Umezeiro proporcionou menor VC, DT, MFP e PPA. Já 'Capdeboscq' e 'Okinawa' proporcionaram à 'Chimarrita' a maior produção acumulada. Para o pessegueiro 'Maciel', os porta-enxertos não influenciaram estatisticamente o VC e o DT, e o portaenxerto Umezeiro proporcionou menor MFP e uma das menores PPA, já que somente o porta-enxerto 'Flordaguard' proporcionou maior PPA (Tabela 2). Na avaliação visual do ponto de enxertia, foi possível observar também um intumescimento nas combinações dos pessegueiros 'Chimarrita' e 'Maciel' com Umezeiro. No local 3 (CAP UFPel), para o pessegueiro 'Chimarrita', o porta-enxerto Umezeiro proporcionou

Tabela 1 - Volume de copa, diâmetro de tronco, massa verde retirada na poda; produção por planta acumulada, comprimento, espessura, número de gemas floríferas e densidade florífera dos ramos produtivos dos pessegueiros 'Chimarrita' e 'Maciel' sobre diferentes porta-enxertos no local 1 (Embrapa Uva e Vinho), valores médios de três anos (2009, 2010 e 2011). FAEM/UFPel, Pelotas/2012.

\begin{tabular}{|c|c|c|c|c|c|c|c|c|}
\hline \multirow[b]{2}{*}{ Porta-enxertos } & \multicolumn{2}{|c|}{----------Planta----------- } & \multirow[b]{2}{*}{$\mathrm{MFP}(\mathrm{Kg})$} & \multirow[b]{2}{*}{ PPA $\left(\mathrm{kg} \mathrm{Pl}^{-1}\right)$} & \multicolumn{4}{|c|}{ - } \\
\hline & $\mathrm{VC}\left(\mathrm{m}^{3}\right)$ & DT (mm) & & & $\mathrm{C}(\mathrm{cm})$ & $\mathrm{E}(\mathrm{mm})$ & $\mathrm{GF}\left(\mathrm{n}^{\underline{\mathrm{o}}}\right)$ & $\mathrm{DF}\left(\mathrm{n}^{\mathrm{o}} / 25 \mathrm{~cm}\right)$ \\
\hline 'Aldrighi'(1) & $1,10 \mathrm{a}$ & $62,05 \mathrm{a}$ & $0,343 b$ & $18,93 \mathrm{c}$ & $26,83^{\mathrm{ns}}$ & $5,29^{\mathrm{ns}}$ & $12^{\mathrm{ns}}$ & $11^{\mathrm{ns}}$ \\
\hline 'Capdeboscq' & $1,603 a$ & $65,39 a$ & $0,255 \mathrm{c}$ & $14,13 d$ & 25,75 & 5,14 & 17 & 17 \\
\hline 'Flordaguard' & $1,231 \mathrm{a}$ & $73,92 \mathrm{a}$ & $0,378 b$ & $19,80 \mathrm{c}$ & 28,19 & 5,48 & 22 & 20 \\
\hline 'Nemaguard' & $1,447 \mathrm{a}$ & $69,81 \mathrm{a}$ & $0,773 \mathrm{a}$ & $35,65 \mathrm{a}$ & 29,47 & 5,39 & 17 & 16 \\
\hline 'Okinawa' & $1,090 \mathrm{a}$ & $67,70 \mathrm{a}$ & $0,361 b$ & $25,65 b$ & 25,03 & 5,12 & 13 & 12 \\
\hline Umezeiro & $0,568 b$ & $47,42 b$ & $0,225 \mathrm{c}$ & $8,70 \mathrm{e}$ & 25,53 & 5,81 & 13 & 12 \\
\hline CV (\%) & 19,70 & 12,48 & 16,36 & 11,74 & 18,9 & 7,01 & 19,98 & 21,77 \\
\hline 'Aldrighi'(2) & $1,51^{\mathrm{ns}}$ & $64,88 \mathrm{a}$ & $0,706 \mathrm{a}$ & $24,80 \mathrm{~b}$ & $36,91^{\mathrm{ns}}$ & $5,98^{\mathrm{ns}}$ & $13^{\mathrm{ns}}$ & $9^{\mathrm{ns}}$ \\
\hline 'Capdeboscq' & 1,332 & $74,93 \mathrm{a}$ & $0,261 b$ & $16,77 \mathrm{c}$ & 37,18 & 5,20 & 19 & 14 \\
\hline 'Flordaguard' & 1,325 & $73,69 \mathrm{a}$ & $0,347 b$ & $9,75 c$ & 34,97 & 5,07 & 24 & 17 \\
\hline 'Nemaguard' & 1,909 & $79,24 a$ & $0,950 \mathrm{a}$ & $33,77 \mathrm{a}$ & 35,97 & 5,62 & 24 & 19 \\
\hline 'Okinawa' & 1,459 & $74,48 \mathrm{a}$ & $0,334 b$ & $13,24 \mathrm{c}$ & 32,47 & 5,34 & 29 & 23 \\
\hline Umezeiro & 1,042 & $49,48 b$ & $0,320 \mathrm{~b}$ & $17,90 \mathrm{c}$ & 30,75 & 5,45 & 20 & 16 \\
\hline $\mathrm{CV}(\%)$ & 26,70 & 13,66 & 31,68 & 17,33 & 26,46 & 10,43 & 12,42 & 18,58 \\
\hline
\end{tabular}

${ }^{(1)}$ Refere-se à cultivar Chimarrita. ${ }^{(2)}$ Refere-se à cultivar Maciel. VC=volume de copa, DT=diâmetro de tronco, MFP=massa fresca da poda, $\mathrm{PPA}=$ produção por planta acumulada, $\mathrm{C}=$ comprimento, $\mathrm{E}=$ espessura, $\mathrm{GF}=$ gemas floríferas, $\mathrm{DF}=\mathrm{densidade}$ florífera. Médias seguidas pela mesma letra minúscula na coluna não diferem entre si pelo teste de Scott e Knott em nível de 5\% de probabilidade de erro. CV (\%) = Coeficiente de variação. ns=não significativo. 
Tabela 2 - Volume de copa, diâmetro de tronco, massa verde retirada na poda e produção por planta acumulada dos pessegueiros 'Chimarrita' e 'Maciel' sobre diferentes porta-enxertos no local 2 (EEA UFRGS), valores médios de três anos (2009, 2010 e 2011). FAEM/UFPel, Pelotas/2012.

\begin{tabular}{llccc}
\hline Porta-enxertos & $\mathrm{VC}\left(\mathrm{m}^{3}\right)$ & $\mathrm{DT}(\mathrm{mm})$ & $\mathrm{MFP}(\mathrm{kg})$ & $\left.\mathrm{PPA}_{(\mathrm{Kg} \mathrm{Pl}}{ }^{-1}\right)$ \\
\hline 'Aldrighi'(1) & $2,594 \mathrm{a}$ & $77,18 \mathrm{a}$ & $2,095 \mathrm{a}$ & $12,12 \mathrm{~b}$ \\
'Capdeboscq' & $2,685 \mathrm{a}$ & $72,84 \mathrm{a}$ & $1,967 \mathrm{a}$ & $17,70 \mathrm{a}$ \\
'Flordaguard' & $2,613 \mathrm{a}$ & $83,37 \mathrm{a}$ & $2,328 \mathrm{a}$ & $12,15 \mathrm{~b}$ \\
'Nemaguard' & $2,102 \mathrm{a}$ & $70,79 \mathrm{a}$ & $2,227 \mathrm{a}$ & $13,40 \mathrm{~b}$ \\
'Okinawa' & $2,778 \mathrm{a}$ & $83,30 \mathrm{a}$ & $2,190 \mathrm{a}$ & $2,529 \mathrm{c}$ \\
Umezeiro & $1,259 \mathrm{~b}$ & $55,60 \mathrm{~b}$ & $0,559 \mathrm{~b}$ & 16,95 \\
CV $(\%)$ & 20,99 & 9,24 & 15,95 & $21,11 \mathrm{~b}$ \\
'Aldrighi' ${ }^{(2)}$ & $3,267^{\mathrm{ns}}$ & $72,76^{\mathrm{ns}}$ & $2,585 \mathrm{a}$ & $23,93 \mathrm{~b}$ \\
'Capdeboscq' & 3,569 & 81,20 & $2,926 \mathrm{a}$ & $30,37 \mathrm{a}$ \\
'Flordaguard' & 3,490 & 82,12 & $3,150 \mathrm{a}$ & $21,28 \mathrm{~b}$ \\
'Nemaguard' & 3,656 & 85,37 & $3,446 \mathrm{a}$ & $19,44 \mathrm{~b}$ \\
'Okinawa' & 3,440 & 76,61 & $2,935 \mathrm{a}$ & $20,64 \mathrm{~b}$ \\
Umezeiro & 2,339 & 63,97 & $1,590 \mathrm{~b}$ & 11,64 \\
CV $(\%)$ & 19,09 & 8,80 & 20,75 & \\
\hline
\end{tabular}

${ }^{(1)}$ Refere-se à cultivar Chimarrita. ${ }^{(2)}$ Refere-se à cultivar Maciel. VC=volume de copa, DT=diâmetro de tronco, MFP=massa fresca da poda, PPA=produção por planta acumulada, $\mathrm{C}=$ comprimento, $\mathrm{E}=$ espessura, $\mathrm{GF}=$ gemas floríferas, $\mathrm{DF}=$ densidade florífera. Médias seguidas pela mesma letra minúscula na coluna não diferem entre si pelo teste de Scott e Knott em nível de 5\% de probabilidade de erro. CV $(\%)=$ Coeficiente de variação. ns=não significativo.

menor VC, DT, MFP, comprimento do ramo produtivo (C) e uma das menores PPA, em que se destacaram os porta-enxertos 'Aldrighi' e 'Flordaguard' com as maiores PPA. Já para o pessegueiro 'Maciel', o portaenxerto Umezeiro proporcionou menor VC, DT, MFP e maior espessura (E), número de gemas floríferas e densidade florífera dos ramos produtivos. A diferença entre os porta-enxertos não foi significativa para PPAe C. Este fato torna possível a utilização do porta-enxerto Umezeiro para o pessegueiro 'Maciel' no local 3 (Tabela 3). $\mathrm{Na}$ avaliação visual do ponto de enxertia, foi possível observar um intumescimento nas combinações dos pessegueiros 'Chimarrita' e 'Maciel' com Umezeiro também no local 3.

Para citros, frequentemente são encontrados porta-enxertos que induzem menor vigor e conferem características interessantes às cultivares copa, assim como ocorreu para o pessegueiro 'Maciel' no local 3, conforme encontrado por CANTUARIAS-AVILÉS et al. (2011), em experimento na região norte do estado de São Paulo, onde o porta-enxerto de Trifoliata 'Flying Dragon' induziu menor vigor e conferiu boas características de produção, possibilitando utilizar o adensamento das plantas.

Avaliando variáveis do desenvolvimento vegetativo, PICOLOTTO et al. (2009) observaram a indução de maior vigor ao pessegueiro 'Chimarrita' com a utilização dos porta-enxertos 'Capdeboscq', 'Tsukuba 1' e 'Okinawa' e baixo vigor com os porta- enxertos 'Aldrighi' e 'GF 305'. O resultado obtido pelos autores contraria os encontrados no presente trabalho, visto que os porta-enxertos 'Aldrighi', 'Capdeboscq' e 'Okinawa' encontram-se no mesmo grupo em relação a aspectos do vigor. Isso se deve ao fato de que os referidos autores não trabalharam com o porta-enxerto Umezeiro, pois este apresenta uma grande divergência dos demais.

A possibilidade de empregar porta-enxertos que induzem menor vigor para a utilização em pomares adensados já é amplamente utilizado na cultura da macieira, podendo também ser utilizado em outras culturas como o pessegueiro. PRAMANICK et al. (2012) julgam que um dos critérios mais pertinentes para a opção de utilização dessa prática é a maximização do rendimento em termos da área do pomar, possibilitando utilizar maior número de plantas por hectare, no entanto, essa condição não deve prejudicar os aspectos fisiológicos da cultura.

Enfim, observou-se que, apesar da variabilidade de comportamento de plantas provenientes de porta-enxertos de semente, o portaenxerto Umezeiro induziu menor vigor nos três locais de cultivo estudados e na média dos três anos. Ele pode ser indicado para utilização sob 'Maciel' nas condições edafoclimáticas do local 3 . No local 1 , o porta-enxerto 'Nemaguard' possui alto vigor e maior produção acumulada, sendo o mais indicado. Já para o local 2, os porta-enxertos 'Capdeboscq', 'Okinawa' e 'Flordaguard' são os mais indicados. 
Tabela 3 - Volume de copa, diâmetro de tronco, massa verde retirada na poda; produção por planta acumulada, comprimento, espessura, número de gemas floríferas e densidade florífera dos ramos produtivos dos pessegueiros 'Chimarrita' e 'Maciel' sobre diferentes porta-enxertos no local 3 (CAP UFPel), valores médios de três anos (2009, 2010 e 2011). FAEM/UFPel, Pelotas/2012.

\begin{tabular}{|c|c|c|c|c|c|c|c|c|}
\hline \multirow[b]{2}{*}{ Porta-enxertos } & \multicolumn{2}{|c|}{---------Planta--------- } & \multirow[b]{2}{*}{ MFP (kg) } & \multirow[b]{2}{*}{ PPA $\left(\mathrm{kg} \mathrm{Pl}^{-1}\right)$} & \multirow[b]{2}{*}{$\mathrm{C}(\mathrm{cm})$} & \multicolumn{3}{|c|}{---------------Ramo produtivo---------------- } \\
\hline & $\mathrm{VC}\left(\mathrm{m}^{3}\right)$ & DT (mm) & & & & $\mathrm{E}(\mathrm{mm})$ & $\mathrm{GF}\left(\mathrm{n}^{\mathrm{o}}\right)$ & $\mathrm{DF}\left(\mathrm{n}^{\mathrm{o}} / 25 \mathrm{~cm}\right)$ \\
\hline 'Aldrighi'(1) & $4,005 \mathrm{a}$ & $76,25 \mathrm{a}$ & $3,206 a$ & $37,28 \mathrm{a}$ & $38,39 a$ & $4,24^{\mathrm{ns}}$ & $17^{\text {ns }}$ & $12^{\text {ns }}$ \\
\hline 'Capdeboscq' & $3,534 \mathrm{a}$ & $78,12 \mathrm{a}$ & $2,924 \mathrm{a}$ & $27,48 b$ & $36,05 \mathrm{a}$ & 4,22 & 17 & 12 \\
\hline 'Flordaguard' & $4,379 \mathrm{a}$ & $83,43 a$ & $3,918 \mathrm{a}$ & $39,80 \mathrm{a}$ & $35,40 \mathrm{a}$ & 4,26 & 17 & 10 \\
\hline 'Nemaguard' & $3,718 \mathrm{a}$ & $72,57 \mathrm{a}$ & $3,075 \mathrm{a}$ & $31,29 b$ & $37,23 \mathrm{a}$ & 4,11 & 17 & 12 \\
\hline 'Okinawa' & $3,179 a$ & $76,71 \mathrm{a}$ & $2,378 \mathrm{a}$ & $30,77 b$ & $35,58 \mathrm{a}$ & 4,17 & 16 & 12 \\
\hline Umezeiro & $2,071 b$ & $61,77 b$ & $1,076 \mathrm{~b}$ & $23,70 \mathrm{~b}$ & $30,53 b$ & 4,32 & 17 & 11 \\
\hline $\mathrm{CV}(\%)$ & 13,49 & 5,66 & 19,53 & 13,27 & 5,90 & 1,43 & 6,57 & 5,18 \\
\hline 'Aldrighi'(2) & $5,034 \mathrm{a}$ & $85,49 a$ & $5,447 a$ & $44,87^{\mathrm{ns}}$ & $46^{\mathrm{ns}}$ & $4,02 \mathrm{~b}$ & $19 b$ & $11 \mathrm{~b}$ \\
\hline 'Capdeboscq' & $4,479 a$ & $82,62 a$ & $4,950 \mathrm{a}$ & 56,49 & 42,57 & $3,96 \mathrm{~b}$ & $18 \mathrm{c}$ & $11 b$ \\
\hline 'Flordaguard' & $5,220 \mathrm{a}$ & $88,44 a$ & $5,876 a$ & 39,89 & 39,27 & $3,87 \mathrm{~b}$ & $16 \mathrm{c}$ & $11 b$ \\
\hline 'Nemaguard' & $5,135 a$ & $84,73 a$ & $5,646 a$ & 54,54 & 43,75 & $3,97 \mathrm{~b}$ & $20 b$ & $12 b$ \\
\hline 'Okinawa' & $4,165 a$ & $85,83 \mathrm{a}$ & $4,346 a$ & 44,35 & 41,44 & $3,85 \mathrm{~b}$ & $17 \mathrm{c}$ & $10 b$ \\
\hline Umezeiro & $2,575 b$ & $65,88 \mathrm{~b}$ & $2,085 b$ & 40,46 & 42,49 & $4,29 \mathrm{a}$ & $24 a$ & $17^{a}$ \\
\hline $\mathrm{CV}(\%)$ & 11,84 & 9,25 & 21,11 & 16,93 & 8,42 & 3,40 & 3,45 & 6,43 \\
\hline
\end{tabular}

${ }^{(1)}$ Refere-se à cultivar Chimarrita. ${ }^{(2)}$ Refere-se à cultivar Maciel. VC=volume de copa, DT=diâmetro de tronco, MFP=massa fresca da poda, PPA=produção por planta acumulada, $\mathrm{C}=$ comprimento, $\mathrm{E}=$ espessura, $\mathrm{GF}=$ gemas floríferas, $\mathrm{DF}=\mathrm{densidade}$ florífera. Médias seguidas pela mesma letra minúscula na coluna não diferem entre si pelo teste de Scott e Knott em nível de 5\% de probabilidade de erro. CV (\%) = Coeficiente de variação. ns=não significativo.

\section{CONCLUSÃO}

Os porta-enxertos afetam as características de crescimento e desenvolvimento das cultivares 'Chimarrita' e 'Maciel', o vigor destas é menor sobre o porta-enxerto Umezeiro nos três locais de cultivo. Todos os porta-enxertos podem ser indicados para os três locais de cultivo, com exceção do Umezeiro, que somente pode ser indicado em combinação com a cultivar Maciel no local 3.

\section{REFERÊNCIAS}

ABDELMAGEED, A.H.A.; GRUDA, N. Influence of grafting on growth, development and some physiological parameters of tomatoes under controlled heat stress conditions. European Journal of Horticultural Science, v.74, p.16-20, 2009. Disponível em: <http://www.ulmer.de/Artikel.dll/ejhs-3907_OTAyMj12.PDF>. Acesso em: 20 dez. 2011.

CANTUARIAS-AVILÉS, T. et al. Horticultural performance of 'Folha Murcha' sweet orange onto twelve rootstocks. Scientia Horticulturae. v.129, p.259-265, 2011. Disponível em: <http:/ /www.sciencedirect.com/science/article/pi / S0304423811001622>. Acesso em: 20 dez. 2011. doi: 10.1016/ j.scienta.2011.03.039.

CRUZ, C.D. Programa GENES - versão windows. Viçosa, MG: Universidade Federal de Viçosa, 2001. 642p. (Versão 2005.0.0).
DE ROSSI, A. et al. Comportamento do pessegueiro 'granada' sobre diferentes porta-enxertos. Revista Brasileira de Fruticultura, v.26, p.446-449, 2004. Disponível em: <http:/ /www.scielo.br/scielo.php?script=sci_arttext\&pid $=$ S010029452004000300018>. Acesso em: 20 dez. 2011. doi: 10.1590/S0100-29452004000300018.

DICHIO, B. et al. Performance of new selections of Prunus rootstocks resistant to Root Knot nematodes, in waterlogging conditions. Acta Horticulturae, v.658, p.403-406, 2004. <http:/ /www.actahort.org/members/showpdf?booknrarnr=658_59>. Acesso em: 5 jan. 2012.

HERNÁNDEZ, F. et al. Performance of Prunus rootstocks for apricot in Mediterranean conditions. Scientia Horticulturae, v.124, p.354-359, 2010. Disponível em: <http://www.sciencedirect.com/ science/article/pii/S0304423810000361>. Acesso em: 23 abr. 2012. doi: 10.1016/j.scienta.2010.01.020.

JIMÉNEZ, S. et al. Influence of different vigour cherry rootstocks on leaves and shoots mineral composition. Scientia Horticulturae, v.112, p.73-79, 2007. Disponível em: <http:/ /www.sciencedirect.com/science/article/pii/ S0304423806004948>. Acesso em: 23 abr. 2012. doi: 10.1016/ j.scienta.2006.12.010.

MARTÍNEZ-BALLESTA, M.C. et al. Review: physiological aspects of rootstock-scion interactions. Scientia Horticulturae, v.127, p.112-118, 2010. Disponível em: <http://www.sciencedirect.com/science/article/pii/ S0304423810003687>. Acesso em: 23 abr. 2012. doi: 10.1016/ j.scienta.2010.08.002. 
MAYER, N.A.; PEREIRA, F.M. Vigor de clones de umezeiro e pessegueiro 'Okinawa' propagados por estacas herbáceas. Pesquisa Agropecuária Brasileira, v.41, p.883-887, 2006. Disponível em: <http://www.scielo.br/scielo.php?script=sci_arttext\&pid=S0100204X2006000500024>. Acesso em: 23 abr. 2012. doi: 10.1590/ S0100-204X2006000500024.

PICOLOTTO, L. et al. Características vegetativas, fenológicas e produtivas do pessegueiro cultivar 'Chimarrita' enxertado em diferentes porta-enxertos. Pesquisa Agropecuária Brasileira, v.44, p.583-589, 2009. Disponível em: <http://www.scielo.br/ pdf/pab/v44n6/a06v44n6.pdf>. Acesso em: 23 abr. 2012. doi: 10.1590/S0100-204X2009000600006.

PRAMANICK, K.K. et al. Performance of apple (Malus x domestica Borkh) cv. 'Red Spur' on a new apple rootstock in high density planting. Scientia Horticulturae, v.133, p.37-
39, 2012. Disponível em: <http://www.sciencedirect.com/ science/article/pii/S0304423811005358>. Acesso em: 23 abr. 2012. doi: 10.1016/j.scienta.2011.10.009.

RATO, A.E. et al. Soil and rootstock influence on fruit quality of plums (Prunus domestica L.) Scientia Horticulturae, v.118, p.218-222, 2008. Disponível em: <http://www.sciencedirect.com/ science/article/pii/S030442380800229X>. Acesso em: 23 abr. 2012. doi: 10.1016/j.scienta.2008.06.013.

USENIK, V. et al. Early detection of graft incompatibility in apricot (Prunus armeniaca L.) using phenol analyses. Scientia Horticulturae, v.109, p.332-338, 2006. Disponível em: <http://www.sciencedirect.com/science/article/pii/ S0304423806002470>. Acesso em: 23 abr. 2012. doi: 10.1016/ j.scienta.2006.06.011. 